P06.03.07

Acta Cryst. (2008). A64, C385

\section{Supramolecular tilt chirality in crystals of cholic acids}

Ichiro Hisaki, Norie 1 Shizuki, Norimitsu 1 Tohnai, Mikiji 1 Miyata Osaka University, Graduate School of Engineering, GSE11F 2-1 Yamadaoka, Suita, Osaka, 565-0871, Japan, E-mail : hisaki@mls.eng. osaka-u.ac.jp

A concept of supramolecular chirality has assumed increasing importance in association with development of supramolecular chemistry over the last two decades. In chiral crystals, 2[1] helical molecular assemblies are frequently observed as key motifs. Helical handedness of the 2[1] assemblies, however, has not been determined from the mathematical, crystallographical viewpoint. In this context, we have proposed a new concept, tilt chirality. On the basis of the concepts, we describe supramolecular chirality and determine the handedness of 2[1] assemblies composed of relatively complicate molecules with multiple stereogenic centres such as bile acids, brucine, and cinchona alkaloids.

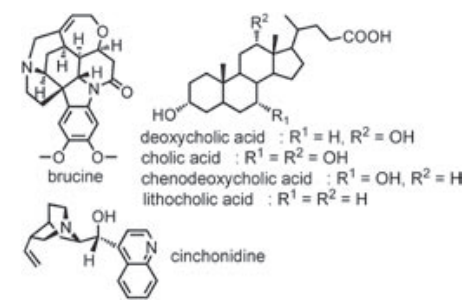

Keywords: alkaloid, helical structure, supramolecular chirality

\section{P06.04.08}

Acta Cryst. (2008). A64, C385

Structure of dihydro-isoindolo benzoxazine-11-one and dibromo-cyclopenta[a]naphthalene-1-one

\section{Mustafa Odabasoglu $^{1}$, Orhan Buyukgungor}

${ }^{1}$ Ondokuz mayis university, OMU Chem. Dep., Kurupelit, SAMSUN, Tr-55139, Turkey, ${ }^{2}$ Ondokuz Mayis University, OMU Physics Dep., Kurupelit, SAMSUN, Tr-55139, Turkey, E-mail:muodabas@omu.edu.tr

Isoindolin-1-one (I) and 2,4-dihydro-1H-benzo[d][1,3]oxazine (II) units are commonly present in synthetic and natural products as simple structures or as a part of complex systems. The syntheses of isoindolin-1-one and 1,3-benzoxazine derivatives have drawn much attention due to the wide range of their biological activities. As part of a continuing study of synthesis of isoindolo-oxazinone and pyrrolooxazinone derivatives and we report here the molecular and crystal structures of (III) and (IV). In the molecule of (III), rings D and C are nearly coplanar with dihedral angle of $2.06(4)^{\circ}$. The dihedral angle between A and D rings is $21.22(3)^{\circ}$. Ring B is not planar and adopts half-chair conformation [1]. In (IV), there are two symmetryindependent molecules in the asymmetric unit and the molecule is not planar, with dihedral angles of $26.5(2)^{\circ}$ and $22.1(2)^{\circ}$ with A and C, respectively. Ring

$\mathrm{B}$ is not planar and adopts half-chair conformation.

[1] Boeyens, J. C. A., J. Cryst. Mol. Struct. 1978, 8, 317-320

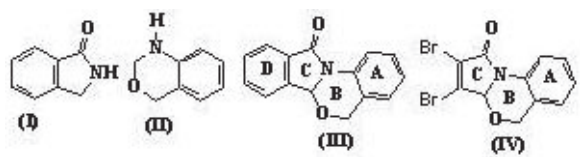

Keywords: isoindoline, benzoxazine, fused ring systems
P06.04.09

Acta Cryst. (2008). A64, C385

\section{Effects of substituents on the oxazaphosphorine heterocyclic compounds}

Krishnaiah Musali ${ }^{1}$, Radha Krishna Jadaprolu ${ }^{2}$

${ }^{1}$ S.V.University, Department of Physics, X-ray crystallography lab, Department of Physics, S.V.University, Tirupati, Andhra Pradesh, 517502, India, ${ }^{2}$ S.V.University, X-ray crystallography lab, Department of Physics, S.V.University, Tirupati, Andhra Pradesh, 517502, India, E-mail : mkphysvu@gmail.com

An increasing interest has been sustained in the chemistry of phosphorus heterocycles owing to their unique physicochemical properties and potential biological activities. Many classes of phosphorus heterocyclic system bearing P-O and P-N units, such as cyclophosphamide and its derivatives, are antitumor agents. The title compounds exhibit antifungal activity against Curvularia lunata. Because of these activities, the X-ray crystal structures shone below are of great interest to our continuing investigations. A comparison between the structures [II \& IV] which differs respectively with substituents of dimethylphenoxy and phenyl at $\mathrm{P}$, shows that the oxazaphosphorine ring assumes a sofa and half-chair conformations. A similar comparison between the compounds [I \& III] exhibits a screw-boat for the former and a boat conformation for the later chlorophenoxy and methoxyphenyl as substituents respectively. The analysis on the above four oxazaphosphorine derivatives, suggests that the substituents at $\mathrm{P}$ are playing a major role on the conformation of the six-membered heterocyclic ring.

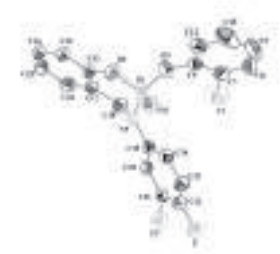

(I)

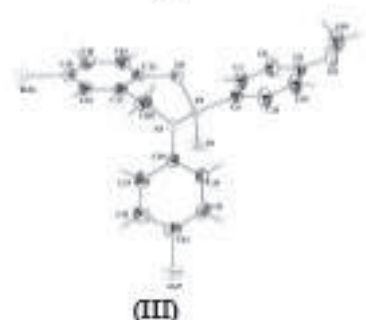

(III)

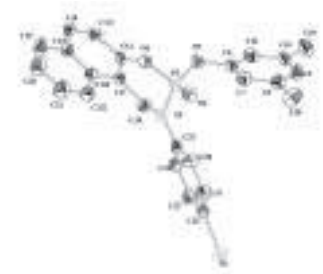

(II)

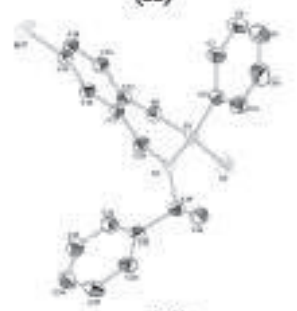

(IV)
Keywords: orgnophosphorus compounds, oxazaphosphorine rings, antifungal agents

\section{P06.04.10}

Acta Cryst. (2008). A64, C385-386

\section{Crystal and molecular structure of 4-7-dimethyl,2-H chromen-2-one} $\frac{\text { Arjuna Gowda K.V. }}{{ }^{1} \text {, Ramohar V Kulkarni }}{ }^{4}$ Rrishna Gowda ${ }^{2}$, Shilpa G M ${ }^{3}$,

${ }^{1}$ Govt. First Grade College, Physics, Department of Physics, Govt. First Grade College, K.R. Pura, Bangalore, Karnataka, 560036, India, ${ }^{2}$ Department of Physics, Govt. College for Women, Kolar, Karnataka, 563101, India, ${ }^{3}$ Department of Physics, Shahyadri College, Shimoga, Karnataka, 577 201,India, ${ }^{4}$ Department of Chemistry, Karnatak University, Dharwad, Karnataka, 580003, India, E-mail:arjunagowda@indiainfo.com 
The title compound belongs to class of Coumarins. Coumarins are toxins found in many plants, notably in high concentration in the tonka bean, woodruff, and bison grass. Coumarines are important because of their extensive plant origin. They have been using in perfume industry. Coumarin and their derivatives have both clinical and medical value as the precursor for several anticoagulants, notably warfarin, used as a gain medium in some dye lasers. The compound $\mathrm{C}_{11} \mathrm{H}_{10} \mathrm{O}_{2}$, crystallizes under orthorhombic system, $P 2{ }_{12}{ }_{1} 2_{1}$ space group, with cell parameters $a=5.232(2), b=11.888(4)$ and $c=$ 13.192(5) $\AA Z=4 \quad V=820.5(5) \AA^{3}$. The data of the compound is collected using Bruker CCD diffractometer with graphite monochromated $\operatorname{MoK} \alpha$ radiation. The structure is solved using SHELXS-97 and refined using SHELXL-97 till R value converges to 0.0714 . There are no intramolecular hydrogen bonds within the molecule. The molecular structure is stabilized by intermolecular $\mathrm{C}-\mathrm{H} . . . \mathrm{O}$ hydrogen bonds and $\mathrm{C}-\mathrm{H} . . . \pi$ interactions.

Keywords: single-crystal structure determination, smallmolecule structures, hydrogen bonds

\section{P06.04.11}

Acta Cryst. (2008). A64, C386

\section{$\mathrm{X}$-Ray data for novel tricyclic compounds based on indolizines}

\section{Victor B. Rybakov ${ }^{1}$, Eugene V. Babaev ${ }^{2}$}

${ }^{1}$ Moscow State University, Chemistry, Vorob'evy Gory, Moscow, none, 199991, Russia, ${ }^{2}$ Moscow State University, Vorob'evy Gory, Moscow, none, 199991, Russia, E-mail:Rybakov20021@yandex.ru

During the past decade we have investigated representative set $(\sim 50$ single crystals and powders) of indolizines I and their precursors - oxazolopyridinium salts II. The structural trends in the families I, II will be overviewed, and the role of the size of third annelated cycle in the salts II on the direction of rearrangement of II to I will be discussed. Novel cyclizations have been found for indolizines III from which novel tricyclic structures IV and V can be formed under the action of bases or acids. Unusual structural features of antiaromatic cyclazines $\mathrm{V}$ will be presented. This work was supported by Russian Foundation for Basic Research (grant 07-03-00921-a).
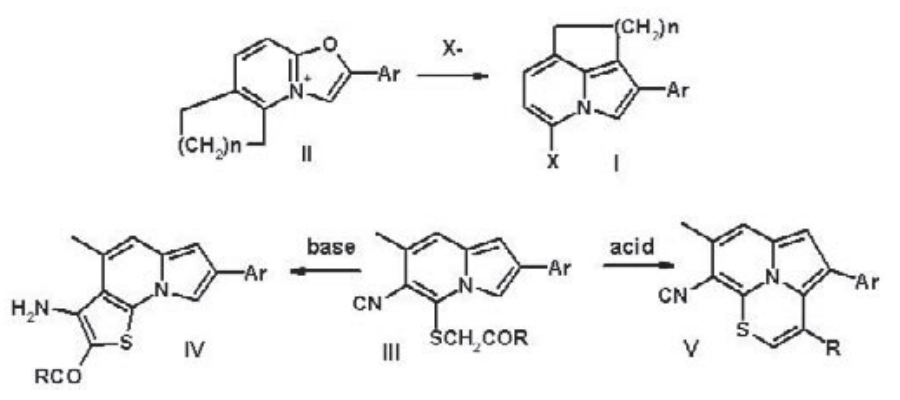

Keywords: molecular structure, heterocycle, indolizine

\section{P06.04.12}

Acta Cryst. (2008). A64, C386

\section{Crystalline molecular assemblies of dehydrobenzo[12]} annulenes having carboxylic groups with amines

Hajime Shigemitsu, Yuu Sakamoto, Ichiro Hisaki, Norimitsu Tohnai, Mikiji Miyata

Division of Advanced Science and Biotechnology, Graduate School of
Engineering, Osaka Universitiy, Department of Material and Life Science, 2-1, Yamadaoka, Suita-City, Osaka, 565-0871, Japan, E-mail : shigemitsu@molrec.mls.eng.osaka-u.ac.jp

Dehydrobenzoannulenes (DBAs) have been of much interest from a viewpoint of their optoelectronic properties due to their rigid, planar, cyclic structures with rich pi-electrons. Moreover, DBA derivatives are adapted as building blocks of supramolecular assemblies. Recently, we reported that hexadehydrobenzo[12]annulene with carboxylic groups form face-to-face, pi-stacked one-dimensional assembly in the crystalline state. The crystal showed significantlyanisotropic charge carrier mobility along the columnar axis, indicating that molecular arrangements play an important role for such properties in the solid state, in addition to molecular structures themselves. Here we present crystal structures of organic salts of octadehydrobenzo[12]annulene having carboxylic groups (Figure) with various amines. The salts were obtained in methanol and then recrystallized from various organic solvents. The hydrogen-bonding groups construct various networks which depend on molecular structures of the amines employed. We discuss a relationship between the molecular arrangements and the optoelectronic properties.

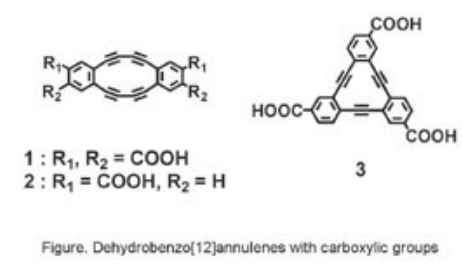

Keywords: molecular assembly, conjugated organic compounds, organic synthesis

\section{P06.04.13}

Acta Cryst. (2008). A64, C386-387

\section{Structures of benzo and dibenzo $[d, f][1,3,2]$ dioxaphosphepine 3-oxide (I, II) and 6-sulphide(III)}

Radha Krishna Jadaprolu $^{1}$, A.K Balaji ${ }^{2}$, Krishnaiah Musali ${ }^{3}$, Suresh Reddy $\mathrm{C}^{4}$, G.Puranik Vedavathi ${ }^{5}$

${ }^{1}$ sri venkateswara university, physics, j.radha krishna, Dept.of physics, s.v.university, tirupati, andhra pradesh, 517502, India, ${ }^{2}$ Dept.of physics, s.v.university, tirupati, andhra pradesh, 517502, India, ${ }^{3}$ Dept.of physics, s.v.university, tirupati, andhra pradesh, 517502 , India, ${ }^{4}$ Dept.of chemistry, s.v.university, tirupati, andhra pradesh, 517502, India, ${ }^{5}$ Physical Chemistry Div., National Chemical Laboratory, Pune, India, E-mail : crystalkrishna@ gmail.com

Orgnophosphorus compounds are widespread in nature and they have unique multifaceted applications as anticancer agents, insecticides and lubricating oil additives and polymer stabilizers. The structures of the title compounds are determined to know the effect of substituents on the conformation of dioxaphosphepine ring. Crystal data (I): $\mathrm{C}_{15} \mathrm{H}_{15} \mathrm{O}_{4} \mathrm{P}$, Monoclinic, space group $P 2_{1} / n, a=9.441(10), b=$ $15.202(16), c=9.746(10) \AA, \beta=95.782(2)^{\circ}, V=1391.5(3) \AA^{3}, Z=$ $4, \mathrm{Dc}=1.385 \mathrm{Mgm}^{-3}, 16173$ reflections measured, 2457 unique [I $>2 \cdot(\mathrm{I})], R$ value $0.0495, \mathrm{w} R 2=0.1145$. Crystal data (II): $\mathrm{C}_{14} \mathrm{H}_{12}$ $\mathrm{Cl} \mathrm{O}_{4} \mathrm{P}$, Monoclinic, space group $P 2_{1} / c, a=13.585(2), b=8.719(1)$, $c=13.106(2) \AA, \beta=118.15(2)^{\circ}, V=1368.7(3) \AA^{3}, Z=4, \mathrm{Dc}=$ $1.508 \mathrm{Mg} \mathrm{m}^{-3}, 6682$ reflections measured, 2410 unique [I $\left.>2 \cdot(\mathrm{I})\right]$, $R$ value $0.0512, \mathrm{w} R 2=0.1118$. Crystal data (III): $\mathrm{C}_{18} \mathrm{H}_{11} \mathrm{Cl}_{2} \mathrm{O}_{3} \mathrm{P}$ $\mathrm{S}$, Monoclinic, space group $P 2_{1} / n, a=10.816(6), b=13.615(8), c$ $=12.321(7) \AA, \beta=99.583(9)^{\circ}, V=1789.0(18) \AA^{3}, Z=4, \mathrm{Dc}=1.519$ $\mathrm{Mg} \mathrm{m}^{-3}, 8553$ reflections measured, 3119 unique [I $\left.>2 \cdot(\mathrm{I})\right], R$ value $0.0428, \mathrm{w} R 2=0.1086$. The dioxaphosphepine rings exhibit twist chair form for I \& II where as distorted boat conformation for III. Fusion of the phosphepine ring to the biphenyl system causes strain, as evidenced by both widening and compression of the endocyclic 